\title{
The role of spatial and temporal contiguity in defensive burying in rats
}

\author{
LYNN ARNAUT and SARA J. SHETTLEWORTH \\ University of Toronto, Toronto, Ontario MSS IAl, Canada
}

\begin{abstract}
The degree of spatial and temporal contiguity between contact with a prod and shock was varied in three experiments to see how these factors contribute to defensive burying. In Experiment 1 , rats shocked once through a grid floor while touching a prod buried the prod just as much as did rats shocked through the prod. Experiment 2 showed that rats either shocked through the floor more than $1 \mathrm{~min}$ after touching the prod or shocked in the absence of a prod did not bury the prod. Thus, close temporal contiguity between grid shock and prod contact appears necessary for burying. Nevertheless, grid-shocked rats do learn something different from prod-shocked rats, since they bury the prod less and the walls more than do prod-shocked rats when the position of the prod is changed in the test chamber (Experiment 3).
\end{abstract}

Hudson (1950) reported that rats tested in an environment with woodshavings often buried shockassociated stimuli after one shock. Pinel and Treit (1978) further investigated the burying response by shocking rats through a prod in a chamber with bedding material covering the floor. Burying the prod was a prominent response in the test chamber. Furthermore, no fleeing, freezing, or fighting was noted. These findings were cited as evidence that burying is a defensive response in an artificial testing environment, and may be a species-specific defensive reaction (SSDR; Bolles, 1970) in an appropriate natural environment. Pinel and Treit suggested that previous failure to observe any SSDRs other than fleeing, freezing, or fighting was due to the constraints of traditional testing environments and not to a lack of other possible responses.

An interesting feature of the conditioned burying paradigm is that burying is observed after only one prod-shock pairing, indicating rapid association of a conditioned stimulus with an unconditioned stimulus. No systematic attempt has been made to account for this in terms of conditioning principles. In order, for example, to label spatial contiguity as a contributing factor in the development of the prod-shock association (e.g., Pinel, Treit, \& Wilkie, 1980), it is necessary to show that decreasing spatial contiguity at the time of conditioning decreased burying. No published studies have included a group in which the CS (the prod) and the US (the shock) were separated

This research was supported by a grant from the Natural Sciences and Engineering Research Council of Canada to the second author. Experiments 1 and 2 were included in a $\mathrm{BSc}$ thesis submitted to the Department of Psychology, University of Toronto, by the first author. We thank S. Fleming for assistance in gathering data and J.P. J. Pinel for sharing his unpublished work. Requests for reprints should be directed to S. J. Shettleworth, Department of Psychology, University of Toronto, Toronto, Ontario MSS 1A 1, Canada. spatially but not temporally. This control is necessary to test the possibility that a shock which is delivered while the rat is touching the prod, but which is not delivered by the prod, may also produce burying.

The role of temporal contiguity between prod contact and shock has also not been tested. Pinel and Treit (1978) shocked rats through one prod while they were in the presence of another prod, and observed no burying of the control prod. However, there has been no control in which subjects have been shocked in the presence of the prod while not in contact with any prod. Terlecki, Pinel, and Treit (1979) have reported that rats buried a novel mousetrap or flashbulb without experiencing any associated aversive stimulus. Thus, since it appears that rats will bury some novel objects without receiving aversive stimulation from them directly, it is important to determine whether burying will occur in the absence of spatial or temporal contiguity between prod contact and shock.

Pinel and Treit (1978) attempted to provide a sensitization control in which rats were shocked through a grid floor with no prod present. The purpose was to demonstrate that a shock alone will not result in unconditional burying of the prod simply as a function of the prod's novelty in the testing environment. They noted that none of the subjects spent a great deal of time burying the prod. However, shock intensity was not identical for the experimental and control groups: grid-shock was $2.5 \mathrm{~mA}$, while the prod-shock was $7.9 \mathrm{~mA}$. Treit, Pinel, and Terlecki (1980) have shown that while a range of prod-shock intensities from .5 to $10 \mathrm{~mA}$ results in burying, animals receiving more intense shock bury more. Thus, an attempt should be made to equate prod and grid shock intensities in order for the absence of a prod in the control group to be the most prominent difference between the two groups. 
The present study varied spatial and temporal contiguity between prod contact and shock in a conditioned burying paradigm. If spatial contiguity is a major factor contributing to conditioning, then rats that receive a shock through the prod should bury the prod more than rats that receive shock from another source. Similarly, if temporal contiguity is important, rats not touching the novel prod during shock should bury the prod less than rats shocked for touching the prod.

\section{EXPERIMENT 1}

In Experiment 1, the importance of spatial contiguity between prod and shock was tested by shocking one group of rats through a prod and a second group of rats through a grid floor while they were touching the prod. Thus, in one group, spatial and temporal contiguity were present between prod contact and shock, while in the other group only temporal contiguity was present.

In this and the following two experiments, all rats were habituated to a test chamber with sawdust on the floor and no prod present. Then they received shock in a second box of the same dimensions but with a grid floor. In this box they could be shocked either through the grid floor or through a prod, which would not have been possible in the first box with the sawdust-covered floor. Finally, rats were tested for the effects of their experience with shock by replacing them in the original test chamber with sawdust and a prod present. Since these aspects of the treatment were the same for all groups, differences in burying in the test can be attributed to differences in their experiences in the shock box.

\section{Method}

\section{Subjects}

The subjects were 16 naive male adult hooded rats obtained from Canadian Breeding Farms and Laboratories (Montreal, Quebec). At the time of testing, they weighed $360-445 \mathrm{~g}$. The rats were housed in individual cages under a 12:12 light:dark cycle. All testing was done in the dark phase of the cycle. The rats had unlimited access to food and water.

\section{Apparatus}

The apparatus consisted of two chambers. The test chamber was a $44 \times 30 \times 44 \mathrm{~cm}$ box. Three sides, the ceiling, and the floor were plywood; the remaining side was Plexiglas. The floor held a $40 \times 29 \times 4 \mathrm{~cm}$ aluminum tray, which contained $4 \mathrm{~cm}$ of sawdust (Betta Chip cage bedding, Maple Leaf Mills, Toronto) at all times. There was an $8 \times 9 \mathrm{~cm}$ hole in the center of each end wall over which $11 \times 11.4 \mathrm{~cm}$ Plexiglas plates could be attached. During habituation, a clear plate was in place at both ends. On the test day, a clear plate with a $6.5-\mathrm{cm}-\mathrm{long} \times 5-\mathrm{cm}$-diam Plexiglas prod wrapped with two uninsulated wires was attached at the left side. The prod was $2.5 \mathrm{~cm}$ above the sawdust. A $7.5-\mathrm{W}$ red light, $33 \mathrm{~cm}$ above the floor in the middle of the back wall, provided the only light in the room.

The shock chamber was identical to the test chamber, with the exception that $324-\mathrm{mm}$ grids were placed $1 \mathrm{~cm}$ apart $4 \mathrm{~cm}$ above the floor. A $40 \times 29 \times 1.7 \mathrm{~cm}$ aluminum tray on the floor under the grids contained $1.5 \mathrm{~cm}$ of sawdust. The prod was in place on the left wall. Either the prod or the grids could be connected to a Grason-Stadler Model 700 shock generator.

A Grason-Stadler noise generator produced $70-\mathrm{dB}$ white masking noise (re .002 dynes $/ \mathrm{cm}^{2}$, measured in the chambers) during all phases of the experiment. The experimenter was present in the room at all times during the experiment and recorded the duration of any burying by pressing a button connected to an EsterlineAngus event recorder and a running iime meter. Session length was measured to the nearest second from the Esterline-Angus tapes, which ran at $7.6 \mathrm{~cm} / \mathrm{min}$.

\section{Procedure}

Habituation. Each rat was placed alone in the test chamber for $15 \mathrm{~min}$ on each of 4 consecutive days, at the same time each day. The purpose of this was to allow the rats to become habituated to the chamber, and for any unconditioned burying to stabilize. After each rat was run, any feces and wet sawdust were removed and the sawdust was leveled out.

On each day, the duration of burying was recorded. Burying was defined as any forward movement or spraying of the sawdust with the forepaws or nose. The experimenter and another observer, separated by a screen, independently recorded burying behavior of rats not used in this experiment for 10 10-min sessions. Their scores for total burying were highly correlated (Spearman rank correlation coefficient $=.94, N=10, p<.01$ ), as were their scores for burying directed toward the prod and elsewhere on the six of those sessions when a prod was present $\left[r_{s}(6)\right.$ $=.943, p<.01]$. Scores for percent total burying directed at the prod correlated perfectly: $r_{s}=1$.

Conditioning and Testing. On the fifth day, the rats were assigned randomly to the prod-shock or the grid-shock group. All rats were individually placed in the shock chamber facing away from the prod, which was on the left wall. When the rats in the prod-shock group first touched the prod with one or both forepaws, they received a $2.5-\mathrm{mA}$ unscrambled shock through the prod. The shock was initiated by the experimenter and terminated by the subject's withdrawal. The rats subsequently remained in the test chamber for $1 \mathrm{~min}$, and then were placed in their home cages for $1 \mathrm{~min}$ while the prod was removed and placed in the test chamber on the left wall. The rats were then placed in the middle of the test chamber facing away from the prod. They remained there for $15 \mathrm{~min}$, and duration of any burying was recorded. Also, the area toward which rats pushed the sawdust was noted; this was called direction of burying.

On Day 5, two of the rats in the prod-shock group failed to touch the prod in the shock chamber within 15 min. On Day 6, they were placed in the chamber again. They were subsequently treated in the same manner as the first six rats.

The eight rats in the grid-shock group were treated in the same manner as the prod-shock group, except that when they first touched the prod with one or both forepaws, they received a .5 -sec 2.5-mA scrambled shock through the grid floor. The shock was initiated by the experimenter. On Day 5, all eight rats in the grid-shock group touched the prod in the shock chamber.

The sawdust was changed after every second or third rat on test days. The grid floor and the prod were cleaned after every rat.

\section{Results and Discussion}

The data consist of the percentage of time spent burying in the test chamber. Percent times were used in place of total times because session times, programmed to be $15 \mathrm{~min}$, varied between 841 and $910 \mathrm{sec}$ among subjects.

Figure 1 shows the median percent time spent burying by both groups on Days 1-5. The percentage 


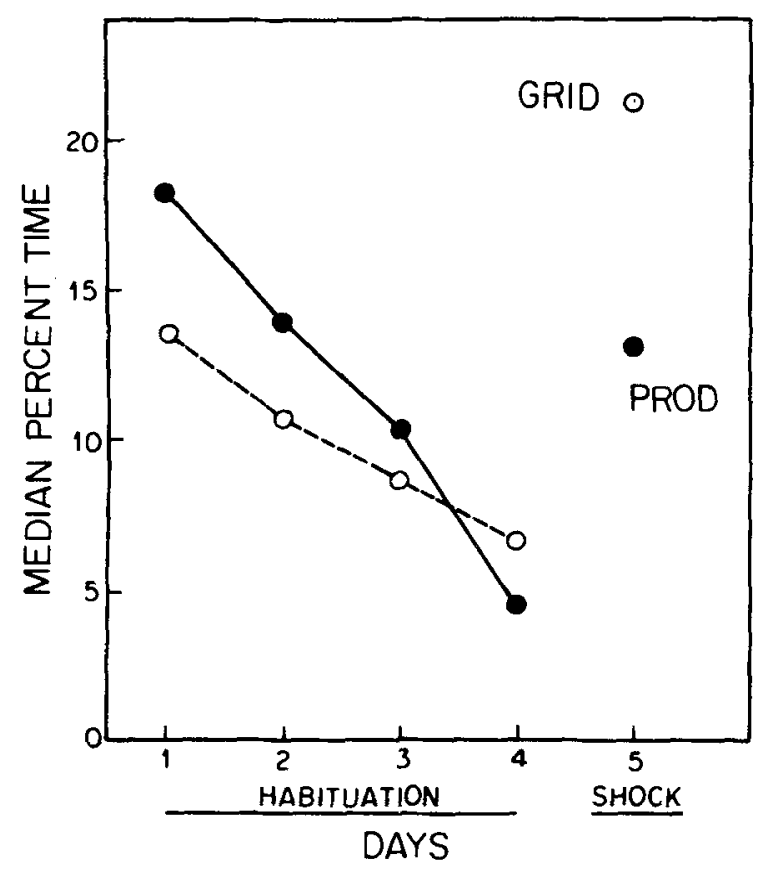

Figure 1. Median percent time burying by prod- and grid-shock groups during habituation and on the test day (Day 5) in Experiment 1.

of time spent burying by each group in the test chamber decreased over the 4 days of habituation. On the test day, both groups increased burying. This change was a significant increase over the last day of habituation for the eight subjects in each group (sign test, ps $<.005$ ).

Virtually all burying in both groups was directed toward the prod on the test day. Four subjects in each group buried the prod completely at least once during testing, but often kept burying, thereby knocking sawdust off the prod. The median height of sawdust piled against the wall at the base of the prod was $10.3 \mathrm{~cm}$ in the prod-shock group and $11.5 \mathrm{~cm}$ in the grid-shock group by the end of the test. Sawdust height at the tip of the prod was 6.8 and $7.3 \mathrm{~cm}$, respectively. Neither measure differed significantly between the groups $[U(8,8) \geqslant 20, p \geqslant .117]$. The percent time spent burying in the test did not differ significantly between groups either $[U(8,8)=20$, $\mathrm{p}=.117]$. No subjects displayed any consistent defensive behavior (e.g., attacking the prod or freezing) other than burying the prod.

Thus, in response to shock, burying was prominent defensive behavior in both groups. It does not appear to be essential for burying that shock come from the object buried, since the grid-shock group buried as much as the prod-shocked group. It should be noted, however, that the two groups may have received qualitatively different shocks. The grid shock was $.5 \mathrm{sec}$ long, while the prod shock was terminated by the subjects. Also, the prod-shock group received the shock through one or both of their forepaws, while the grid-shock group received it through their hindpaws. Thus, shock duration, intensity, and/or quality may have varied between groups and, consequently, the aversiveness of the shock may have varied as well. In conclusion, then, the important result of the present experiment is simply that substantial burying did occur when shock was received through the grid floor.

\section{EXPERIMENT 2}

Given that spatial contiguity of CS and US typically facilitates conditioning (Rescorla \& Cunningham, 1979), it is somewhat surprising that rats shocked through the floor while touching the prod in Experiment 1 buried the prod as much as did rats shocked through the prod itself. This finding suggests that perhaps burying in Experiment 1 was not the result of association between prod and shock, but rather a sensitized response to the novel prod in the test chamber. Alternatively, placement in the shock chamber, together with shock, and perhaps also the appearance of the novel prod in the test chamber could be viewed as dishabituating the partially habituated burying response. This possibility is consistent with the finding that burying in the test was not greater than burying in the first session of habituation (see Figure 1). Such an account, however, does not immediately explain why most burying in the test was directed towards the prod.

Experiment 2 was designed to test the role of temporal contiguity between prod contact and grid shock in the development of burying. Rats were shocked through the grid floor approximately $1 \mathrm{~min}$ after they first touched the prod. A sensitization control group was included in which rats were shocked with no prod present, in order to judge whether shock alone would lead to burying of the novel prod in the test chamber. All rats were shocked when rearing in order to equate the shock with that received by the grid-shock animals in Experiment 1 which were shocked through the hindpaws while rearing to touch the prod.

\section{Method}

\section{Subjects and Apparatus}

The subjects were 16 naive male adult hooded rats like those in Experiment 1. The apparatus was the same as that described previously.

Procedure

Habituation. All 16 subjects received 4 days of habituation as described in Experiment 1.

Conditioning and Testing. On the fifth day, the rats were assigned randomly to one of two groups. The eight rats in the first group (Group 1) were placed individually in the shock chamber facing away from the prod. The time before each rat touched the prod once with one or both forepaws was recorded. Sub- 
sequently, after $1 \mathrm{~min}$ had passed, the rats received a .5 -sec 2.5-mA scrambled shock through the grid floor the first time they reared and were not in contact with the prod. The location of shock delivery in the chamber was also recorded.

Each rat in the second group (Group 2) was matched to one of Group 1's times between placement in the chamber and first prod contact. They were individually placed in the shock chamber with no prod present. After the matched time plus $1 \mathrm{~min}$ had elapsed, they were shocked the first time they reared. After receiving shock, all rats were observed in the test chamber, as in Experiment 1.

\section{Results and Discussion}

No consistent burying was observed in either group. The data of three rats were discarded due to procedural difficulties. On the test day, neither group showed a significant increase in burying over habituation (sign test, $p>.05$ ). Median percent times burying were 10.7 in Group 1 and 4.2 in Group 2; the groups did not differ significantly on this measure $[\mathrm{U}(7,6)=15, \mathrm{p}=.223]$.

Two of the seven rats in Group 1 (grid shock with prod present) and one of the six rats in Group 2 (no prod present) pushed sawdust toward the prod. The behavior of the remaining 10 rats was very variable. One subject in Group 1 and two in Group 2 did no burying at all; one in each group froze in the corner opposite to where it had received shock in the shock chamber, and the other rat walked around. The remaining subjects pushed sawdust toward the walls and corners. Four rats in Group 1 and two in Group 2 piled sawdust in the location corresponding to where they had received shock in the shock chamber. The median depths of sawdust at the base of the prod at the end of the test were $4.6 \mathrm{~cm}$ in Group 1 and $5.0 \mathrm{~cm}$ in Group 2; at the tip, they were 4.0 and $5.3 \mathrm{~cm}$, respectively [Us $(7,6)$ $>16$, ps $>.25]$. The values are close to the $4.0-\mathrm{cm}$ depth at the beginning of the test and much less than those in Experiment 1.

Thus, no consistent defensive behaviors were exhibited by either group. In particular, the absence of reliable burying of the prod in Group 1 suggests that temporal contiguity between prod contact and shock is necessary for burying to occur. Group 2, shocked with no prod present, did not bury either, indicating that neither shock alone nor the novelty of the prod in the test chamber after shock in the grid box results in burying of the prod. Thus, in Experiment 1 , the burying exhibited by the rats shocked through the grid while touching the prod must represent associative learning of some sort.

\section{EXPERIMENT 3}

In Experiment 1, all rats buried the prod, whether they had been shocked by the prod or the grid floor. In Experiment 2, 6 of 10 rats that had been shocked through the grid and had displayed any burying appeared to direct their burying toward the area in the test chamber that corresponded to the location of shock delivery in the shock chamber. Pinel et al. (1980) found that, when the position of a shock prod was changed at the time of testing, rats often buried the hole where the prod had been originally, even though the prod was present at the other hole. Hudson (1950) reported a similar observation. Thus, the rats in Experiment 1 may have buried the prod not only because it was associated with shock, but also because it was in the location of shock delivery. It is possible that the prod-shock subjects formed a more specific prod-shock association than the grid-shock subjects, which, in turn, may have learned more about cues associated with shock location. In Experiment 3, the procedure was identical to that of Experiment 1 except that the prod location was changed at the time of testing. If learning is different in the presence of spatial contiguity, then the prod-shock subjects may spend more time burying the prod.

\section{Method}

\section{Subjects and Apparatus}

The subjects were 16 naive male adult hooded rats like those in Experiment 1. The apparatus was the same as that described previously.

\section{Procedure}

Habituation. All 16 subjects received 4 days of habituation, as in Experiment 1. No measures were taken during this time.

Conditioning and Testing. On Day 5 , the rats were randomly assigned to a prod- or a grid-shock group, with eight in each group. Rats in both groups were placed in the shock chamber and shocked through either the prod or the grid floor when they touched the prod, which was always in the left wall. The procedure was the same as that in Experiment 1, with two exceptions. First, when the prod was removed and placed in the test chamber, it was placed on the right wall. Second, the duration of burying directed towards the prod was recorded, as was the duration of burying in any other direction. The direction of burying was judged by where the rat pushed the sawdust. These measures combined to provide a total burying duration.

On the test day, three of the rats in the prod-shock group failed to touch the prod in the shock chamber within $15 \mathrm{~min}$. The rats were removed and placed in the shock chamber again on Day 6. Two rats did not touch the prod on Day 6 , and thus no data are reported for these subjects. The third rat touched the prod and was subsequently treated in the same manner as the other five rats.

\section{Results and Discussion}

The total percentage of time spent burying on the test day did not differ between the two groups [MannWhitney $\mathrm{U}(6,8)=22, \mathrm{p}>.05$ ]. The range of scores was from $5.48 \%$ to $44.76 \%$, which was similar to that found in Experiment 1 (4.47\% to $48.69 \%$ ).

The prod-shock group spent significantly more time burying the prod than did the grid-shock group (median test, $\mathrm{p}<.05$ ). Table 1 shows the median percentage of burying directed at the prod by both groups. The scores of 5 of the 6 rats in the prodshock group were above the median prod-directed 
Table 1

Results of Experiment 3

\begin{tabular}{lrr}
\hline & \multicolumn{2}{c}{ Shock } \\
\cline { 2 - 3 } & Prod & Grid \\
\hline Median Percent of Test Session Burying & 6.0 & 8.0 \\
Median Percent of Burying Directed at Prod & 87.3 & 59.9 \\
\hline
\end{tabular}

burying score for all 14 rats. The 6th rat did not bury the prod at all; rather, he appeared to burrow underneath the sawdust during the test.

Most of the burying not directed toward the prod by the grid-shocked rats was directed at the walls of the box; only three of the eight subjects specifically buried the left wall (the original prod location). At the end of the test, depths of sawdust at the prod did not reflect the differences in overall burying behavior of the two groups. The median depths at the base were $7.0 \mathrm{~cm}$ in the prod-shock group and $8.0 \mathrm{~cm}$ in the grid-shock group; at the tip, the depths were 6.0 and $6.5 \mathrm{~cm}$, respectively [Us $(8,6)>.28]$.

The observations of where burying was directed show that prod-shocked and grid-shocked rats do learn different things. In particular, rats shocked through the prod seem to form a more specific prodshock association than do rats shocked through the grid floor while touching the prod. Pinel et al. (1980) noted that when a prod was removed from a hole and placed on the opposite wall, several prod-shocked rats pushed sawdust toward the hole as well as toward the prod. In another experiment, when the positions of a black prod and a white prod were interchanged, the rats directed burying toward both prods, that is, toward both the brightness and the location of the prod that had shocked them. In the present case, grid-shocked rats directed some of their burying toward the prod and some toward other parts of the box, but they did not push sawdust only toward the part of the test chamber corresponding to where they had been shocked, nor did prod-shocked rats direct much burying at the old prod location. Here, however, the source of shock was not well defined by cues other than the prod itself, especially because the test occurred in a different box from shock. The shock box was similar to the familiar test box, too, which may have impeded associating with shock a specific part of the box other than the novel prod or the novel grid (absent in the test chamber) with shock. These factors may at least partly account for why burying by the grid-shocked rats in the present experiment was rather diffuse.

\section{GENERAL DISCUSSION}

For defensive burying directed at a prod to occur, shock need not be delivered through the prod. Gridshocked rats in Experiment 1 buried the prod as much as did rats shocked by the prod, indicating that rats can associate a floor shock with the prod. It should be noted that there was some spatial contiguity between prod and grid shock, since the gridshocked rats were standing next to the prod and touching it. Although, in Experiment 2, the rats that were near the prod at the time of shock did not bury the prod more than rats shocked elsewhere in the shock chamber, it remains to be seen whether rats must be touching the prod at the time of shock to bury it reliably.

The results of Experiment 3 suggest that gridshocked rats did not form as specific a prod-shock association as did the prod-shocked subjects. However, they did not consistently bury the old prod location. Perhaps the grid-shocked rats learned about cues other than location which were not accounted for in the present experiment; for example, they may have learned that the grid floor delivers shock.

Alternatively, if the novel prod was the most salient stimulus present, the shock location may have been most well defined by the prod, and thus other cues were ignored. If other cues could easily be associated with shock location, then the grid-shocked rats shocked with the prod present in Experiment 2 should have displayed more consistent burying of shock location than they did. Thus, perhaps the rats do learn about location, but in the present experiment they may have learned that the prod defines the location. If the rats were presented with another salient stimulus in addition to the prod (e.g., a pattern) which they could associate with the original shock location, they may show more burying directed toward the location itself, instead of the prod-directed and general burying observed in Experiment 3. Hudson (1950) had his shock source (a food dish) surrounded by a distinctive novel pattern, but he did not report any studies of burying directed at the pattern itself in different locations.

Experiment 2 demonstrated that neither a shock in the presence of the prod after contact nor a shock alone is sufficient to produce prod-directed burying. The finding that temporal contiguity is important in the development of defensive burying is consistent with previous research. Most reports of conditioning with exteroceptive stimuli have reported decreasing responses with increasing CS-US intervals. In general, only paradigms using illness to produce an aversion to a flavor have been able to produce conditioning with interstimulus intervals of over several seconds or, at most, minutes (Mackintosh, 1974).

An interesting exception to the general findings concerning the importance of temporal contiguity in the association of shock with exteroceptive stimuli is a study by Sullivan (1979). She found long-delay onetrial learning in rats, using a paradigm which paired touching a novel object with a shock $\mathbf{3 0}$ min later, in an environment to which the rats had been habituated. 
One possible reason why long-delay learning was not exhibited in Experiment 2 may have been that, although the shock chamber was objectively identical to the test chamber, with the exception of the grid floor and the prod, it may still have constituted a novel environment to the rats. Perhaps long-delay learning would be exhibited if the animals had been habituated to the shock chamber as they had been to the test chamber before shock. Thus, the prod would be the only novel stimulus present on the shock day, and may consequently be more easily associated with a temporally separate shock.

In conclusion, the conditioned burying paradigm (Hudson, 1950; Pinel \& Treit, 1978) is of interest partly because the contingency between contact with an object and aversive stimulation from the object appears to mimic a situation which must commonly occur in nature. Thus, just as with conditioned taste aversion, it seems reasonable that the resulting learning should occur rapidly. We have shown that the association underlying burying is equally rapid when the aversive stimulus does not come from the object buried, even though rats do behave differently in some situations, depending on where the aversive stimulus originated (Experiment 3). Thus, the present experiments begin to define how rats identify objects as sources of aversive stimuli.

\section{REFERENCES}

Bolles, R. C. Species-specific defense reactions and avoidance learning. Psychological Review, 1970, 77, 32-48.

Hudson, B. B. One-trial learning in the domestic rat. Genetic Psychology Monographs, 1950, 41, 99-145.

Mackintosh, N. J. The psychology of animal learning. London: Academic Press, 1974.

Pinel, J. P. J., \& Treit, D. Burying as a defensive response in rats. Journal of Comparative ana Physiological Psychology, 1978, 92, 708-712.

Pinel, J. P. J., Treit, D., \& Wilkie, D. M. Stimulus control of defensive burying in the rat. Learning and Motivation, 1980, 11, 150-163.

Rescorla, R. A., \& Cunningham, C. L. Spatial contiguity facilitates Pavlovian second-order conditioning. Journal of Experimental Psychology: Animal Behavior Processes, 1979, 5, 152-161.

Sullivan, L. Long-delay learning with exteroceptive cue and exteroceptive reinforcement in rats. Australian Journal of Psychology, 1979, 31, 21-32.

Terlecki, L. J., Pinel, J. P. J., \& Treit, D. Conditioned and unconditioned defensive burying in the rat. Learning and Motivation, 1979, 10, 337-350.

Treit, D., Pinel, J. P. J., \& Terlecki, L. J. Shock intensity and conditioned defensive burying in rats. Bulletin of the Psychonomic Society, 1980, 16, 5-7.

(Received for publication July 2, 1980; revision accepted December 2,1980 .) 